\title{
Spectral flow of non-hermitian Heisenberg spin chain with complex twist
}

\author{
Takahiro Fukuit \\ Institute of Advanced Energy, Kyoto University, Uji, Kyoto 611, Japan \\ Norio Kawakami \\ Department of Applied Physics, Osaka University, Suita, Osaka 565, Japan
}

(September 29, 1997)

We investigate the spectral flow of the integrable nonhermitian Heisenberg spin chain under boundary conditions with complex twist angle. It is shown that the period of the spectral flow is $4 \pi$ up to a certain critical imaginary twist, beyond which the period jumps successively to higher values. We argue that this phenomenon caused by nonhermitian properties of the system is closely related to the metal-insulator transition caused by non-hermitian hoppings for the one-dimensional insulator.

PACS numbers: 75.10.Jm, 05.30.-d

Keywords: Asymmetric Heisenberg model, Complex twisted boundary condition, Non-hermitian Hamiltonian, Spectral flow, Bethe ansatz method, Finite size correction

\section{INTRODUCTION}

Boundary conditions have been playing an important role in statistical mechanics. Imagine for instance a one-dimensional (1D) many particle system with twisted boundary conditions where a particle gets additional phase factor $e^{i \Phi}$ when it moves once along the system with a ring geometry and return to the previous position. For integrable systems, this general phase factor is simply incorporated into the Bethe ansatz equations, and their characteristic properties have been intensively studied exactly [1] condition is equivalent to introducing a magnetic flux threaded in the ring system [5]. In proportion as the flux increases, the spectrum of the system flows in a complex way, experiencing many level crossings and level repulsions. We can get detailed information for the physical properties from the spectral flow thus obtained. For example, a local behavior of the spectral flow near $\Phi \sim 0$ directly gives the transport coefficient such as the Drude weight, which is a sensitive probe of the metal-insulator transition [6,7]. From a more global structure of the spectral flow 810], especially paying attention to its period for the ground state, one can also determine whether or not a bound state forms [11, 12]. Berry's phase was calculated in this process [13][14], which may carry the information for statistics of elementary excitations. It was indeed shown $15 \sqrt{17}$ that the period of the spectral flow is directly related to Haldane's fractional exclusion statistics [18]. Also, the twisted boundary condition has attracted other current interests 19,20].

Normal twisted boundary conditions can be further generalized to those with complex twist angles, $\Phi \rightarrow$ $\Phi+i \Psi$ [3]. They make the Hamiltonian non-hermitian, which is the most remarkable difference from normal twisted boundary conditions. The spectral flow is now defined on the complex plane and shows a rich structure. An interesting point to be emphasized is that this problem has the close relationship to recent topics studied actively in the context of non-hermitian systems. In particular, it was claimed by Hatano and Nelson 21] that introducing non-hermitian hopping terms into the Hamiltonian brings about the localization-delocalization transition even for 1D random systems. The key point is that eigenvalues become complex if we increase the imaginary twist angle, which can provide a criterion whether the delocalization happens. Many interesting problems on the non-hermitian Hamiltonian systems still remain to be explored. In order to study such problems, integrable non-hermitian models are quite useful.

In this paper, we investigate a $1 \mathrm{D}$ non-hermitian system with twisted boundary conditions, and discuss how the spectral flow changes its character if the imaginary twist is introduced. As a typical example, we examine a version of the asymmetric Heisenberg spin chain and the related vertex models studied by [22 30]. It was argued that asymmetry causes interesting phenomena. For example, by introducing non-hermitian XY components, the massive XXZ spin chain with Ising-type anisotropy is driven to the massless phase, which is regarded as a kind of the metal-insulator transition [29. The model we will study in this paper is the isotropic Heisenberg spin chain, which is massless even for zero imaginary twist. However, if we consider a finite-size system, there exist many small gaps due to finite-size effects. It is these gaps that determine the period of the ground state in the global spectral flow. We show that introducing the non-hermitian imaginary twist may collapse these gaps, changing the period to a larger one. It is argued that this phenomenon has essentially the same origin as for the bulk metal-insulator transition in 1D non-hermitian many-body systems: In both cases, the transition is triggered by complex eigenvalues due to non-hermitian hopping terms.

This paper is organized as follows. In the next section, we introduce the model and the Bethe ansatz equations, and briefly summarize the characteristics in the spectral 
flow of the ordinary symmetric (hermitian) model. In section III, we discuss the spectral flow of the asymmetric model with complex twist angles, by numerically evaluating the Bethe-ansatz spectrum for small systems. We show that there is indeed the critical value of complex twist, at which the period of the spectral flow suddenly changes. In IV, we estimate this critical value for the transition for large but finite-size systems. For this purpose, we propose an approximate treatment to obtain the finite-size corrections rather accurately. Final section V is devoted to summary and discussions.

\section{MODEL AND BASIC PROPERTIES OF THE SPECTRAL FLOW}

In this section, we introduce the model and summarize some basic properties of the spectral flow for the ground state of the hermitian (symmetric) model.

\section{A. Model and Bethe ansatz equations}

The model we consider is the well-known isotropic Heisenberg spin chain

$$
H=\sum_{j=1}^{N}\left(\boldsymbol{S}_{j} \cdot \boldsymbol{S}_{j+1}-\frac{1}{4}\right),
$$

but with the twisted boundary condition,

$$
S_{j+N}^{ \pm}=e^{ \pm i \Theta} S_{j}, \quad S_{j+N}^{z}=S_{j}^{z} .
$$

The spectral flow has been discussed so far for real $\Theta$. What we are now interested in is how the ground state behaves for the complex twist angle,

$$
\Theta=\Phi+i \Psi,
$$

which makes the Hamiltonian (2.1) non-hermitian. In the case of purely imaginary twist angle, the model is the same as the asymmetric Heisenberg chain but with the replacement $\Psi \rightarrow N \Psi$. The asymmetric XXZ Heisenberg chain has been studied so far in the massive regime, while our isotropic model is in the massless regime for which the non-hermitian effects disappear in the thermodynamic limit. We shall in turn treat the model for a finite-size system, and discuss how the non-hermitian property affects its spectral flow by examining the $1 / N$ corrections.

We begin by summarizing the symmetry properties of the model. Define the eigenvalue problem as

$$
\begin{aligned}
H(\Theta)|\psi(\Theta)\rangle_{R} & =E_{R}(\Theta)|\psi(\Theta)\rangle_{R} \\
{ }_{L}\langle\psi(\Theta)| H(\Theta) & ={ }_{L}\langle\psi(\Theta)| E_{L}(\Theta)
\end{aligned}
$$

Because of the symmetries $H^{*}(\Theta)=H\left(-\Theta^{*}\right), H^{\dagger}(\Theta)=$ $H\left(\Theta^{*}\right)$, and $U H(\Theta) U^{\dagger}=H(-\Theta)$ with $U=e^{-i \pi \sum S_{j}^{y}}$ we have

$$
\begin{aligned}
& \left|\psi\left(-\Theta^{*}\right)\right\rangle_{R}=\left|\psi^{*}(\Theta)\right\rangle_{R}, \quad E_{R}\left(-\Theta^{*}\right)=E_{R}^{*}(\Theta), \\
& \left|\psi\left(\Theta^{*}\right)\right\rangle_{R}=U\left|\psi^{*}(\Theta)\right\rangle_{R}, \quad E_{R}\left(\Theta^{*}\right)=E_{R}^{*}(\Theta), \\
& |\psi(\Theta)\rangle_{L}=U\left|\psi\left(-\Theta^{*}\right)\right\rangle_{R}, \quad E_{L}(\Theta)=E_{R}(\Theta) .
\end{aligned}
$$

As shown by Yung and Batchelor [3], the model is solved by the Bethe ansatz method,

$$
N p\left(\lambda_{j}\right)+\sum_{k \neq j}^{M} \theta\left(\lambda_{j}-\lambda_{k}\right)=2 \pi I_{j}+\Theta, \quad j=1, \cdots, M,
$$

where the momentum $p(\lambda)$ and the two-body phase shift $\theta(\lambda)$ as functions of the rapidity $\lambda$ are given by

$$
\begin{aligned}
& p(\lambda)=-i \ln \left(-\frac{\lambda-i / 2}{\lambda+i / 2}\right), \\
& \theta(\lambda)=i \ln \left(-\frac{\lambda-i}{\lambda+i}\right) .
\end{aligned}
$$

Here the branch cut lies on $(-i \infty,-i / 2)$ and $(i / 2, i \infty)$ for $p(\lambda)$ and on $(-i \infty,-i)$ and $(i, i \infty)$ for $\theta(\lambda)$. The total energy of the system is given by the summation of single particle energies,

$$
E=\sum_{j=1}^{M} \frac{-2}{4 \lambda_{j}^{2}+1} .
$$

In the rest of the paper, we concentrate on the spectral flow of the ground state, so that we set $M=N / 2$ with $N=$ even, and

$$
I_{j}:-\left(\frac{N}{4}-\frac{1}{2}\right), \cdots,\left(\frac{N}{4}-\frac{1}{2}\right) .
$$

\section{B. Spectral flow for the case $\Psi=0$}

Before we proceed to the non-hermitian model, let us briefly summarize the spectral flow of the model with an ordinary hermitian condition $\Psi=0$. This case was investigated in detail by [2, 8, 9, 13,14], who claimed that the ground state becomes the first excited state at $\Phi=2 \pi$ and returns to the ground state again at $\Phi=4 \pi$, namely, the period of the ground state is $4 \pi$ as a function of $\Phi$. This feature can be observed indeed in Fig. 1, which is obtained by the exact diagonalization for the $S^{z}=0$ sector of the $N=6$ system. The period $4 \pi$ is caused by the small gaps made by level repulsions due to irrelevant backward interactions, and is quite general for ordinary finite-size systems with such irrelevant interactions. This 
property will be essentially changed by the introduction of non-hermitian terms, as is discussed in the next section.

Before concluding this section, we wish to give an interpretation of the above spectral flow in terms of the Bethe-ansatz language [8,14. At $\Phi=0$ the $N$ rapidities for the ground state are distributed symmetrically on the real axis. As $\Phi$ approaches $2 \pi$, the last rapidity $\lambda_{M}$ runs to infinity, while the others shift a little bit to the positive direction, and are arranged in a configuration of the lowest energy state for the $S^{z}=1 / 2$ sector. This can be seen clearly if we divide the Bethe ansatz equations (2.6) at $\Phi=2 \pi$ into two classes,

$$
N p\left(\lambda_{j}\right)+\sum_{k \neq j}^{M-1} \theta\left(\lambda_{j}-\lambda_{k}\right)+\theta\left(\lambda_{j}-\lambda_{M}\right)=2 \pi\left(I_{j}+1\right),
$$

for $j=1, \cdots, M-1$ and

$$
N p\left(\lambda_{M}\right)+\sum_{k=1}^{M-1} \theta\left(\lambda_{M}-\lambda_{k}\right)=2 \pi\left(I_{M}+1\right),
$$

The last equation is satisfied by $\lambda_{M}=+\infty$, and the former equations reduce to

$$
N p\left(\lambda_{j}\right)+\sum_{k \neq j}^{M-1} \theta\left(\lambda_{j}-\lambda_{k}\right)=2 \pi I_{j}^{\prime}
$$

where

$$
I_{j}^{\prime}:-\frac{N}{4}, \cdots, \frac{N}{4} .
$$

Beyond $\Phi=2 \pi$, the rapidity $\lambda_{M}$ jumps from infinity to minus infinity and at last all rapidities return to the original configuration at $\Phi=4 \pi$. In this process, the rapidity $\lambda_{M}$ crosses the branch cut of the logarithmic function in eq.(2.7), so that

$$
\begin{aligned}
& p\left(\lambda_{j}^{(4 \pi)}\right)=p\left(\lambda_{j+1}^{(0)}\right), \quad j=1, \cdots, M-1, \\
& p\left(\lambda_{M}^{(4 \pi)}\right)=p\left(\lambda_{1}^{(0)}\right)+2 \pi .
\end{aligned}
$$

This gives an interpretation of the period $4 \pi$, which is due to irrelevant interactions, in terms of the motion of the rapidities. In the next section, by extending the above idea, we discuss how the spectral flow changes its character if we switch on the imaginary twist $\Psi$.

\section{SPECTRAL FLOW FOR THE NON-HERMITIAN MODEL}

\section{A. Basic properties of the spectral flow}

We now extend the twist angle to a complex one. We should hence consider the spectral flow on various paths on the complex $\Theta$ plane. In order to see clearly how the spectral flow occurs, let us regard the flow as a function of $\Phi$ with a fixed $\Psi$. For small $\Psi$, the flow is essentially the same as the $\Psi=0$ case, as can be seen in Fig. 2, where only the real part of E's is plotted for simplicity. Namely, except for the spectrum being complex, the qualitative nature of the whole spectral flow is not modified by a finite but sufficiently small $\Psi$. This implies that such small $\Psi$ cannot collapse the $1 / N$ gaps due to irrelevant backward scatterings. It should be noted that at $\Phi=2 \pi(\bmod 2 \pi)$ all eigenvalues are real. If $\Psi$ is further increased, the gap between the first and the second excited state becomes small and eventually disappears at a certain $\Psi=\Psi_{\mathrm{cr}}$, above which these two states become degenerate in the real part of $E$ (They are complex conjugate each other). Then the period of the ground state changes, as seen in Fig. 3. In this case, the period of the ground state is $8 \pi$. If we further increase $\Psi$, the period becomes longer. For example, in Fig. \&4 it becomes macroscopic value $2 \pi N(=12 \pi)$. Physically, the change of the period means that the small $1 / N$ gaps due to irrelevant interactions are successively closed by the nonhermitian effects, as mentioned above. This phenomenon has the essentially same origin as for the metal-insulator transition triggered by non-hermitian hoppings.

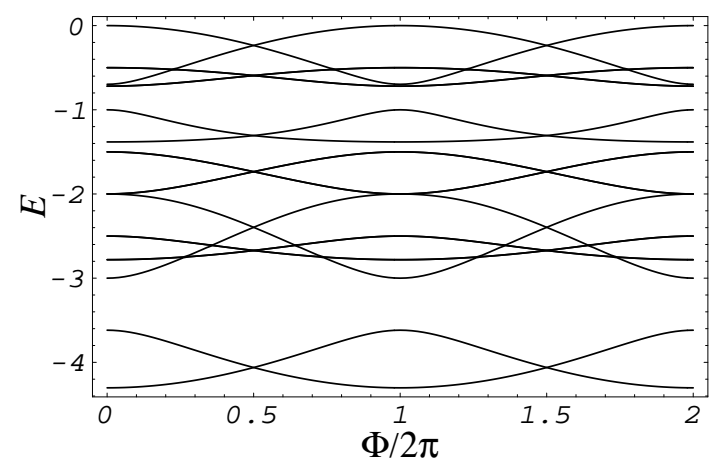

FIG. 1. Spectral flow of the $S^{z}=0$ sector of the $N=6$ with $\Psi=0$ system obtained by the exact numerical diagonalization

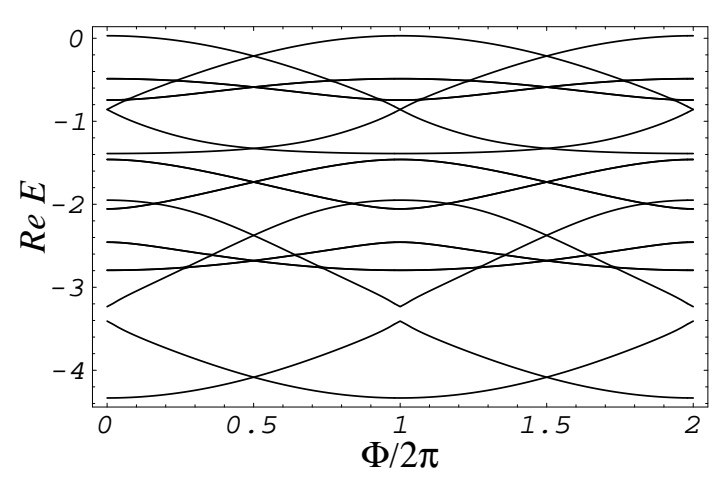

FIG. 2. The same spectral flow but with $\Psi=1.1$

Let us now consider the spectral flow defined on the whole complex $\Theta$ plane, which is sketched qualitatively 


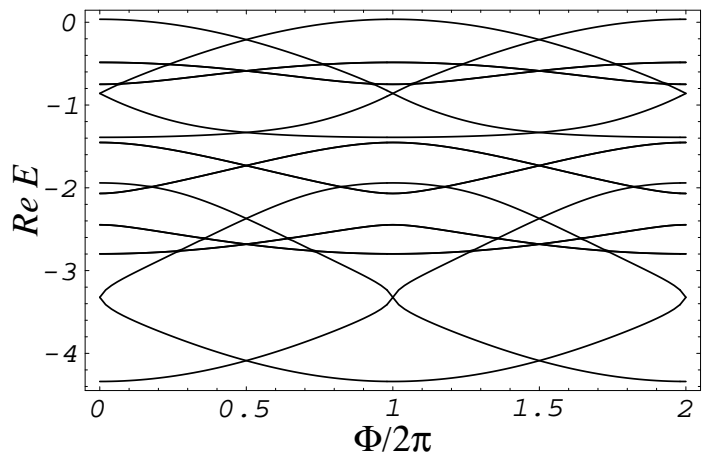

FIG. 3. The same spectral flow but with $\Psi=1.2$

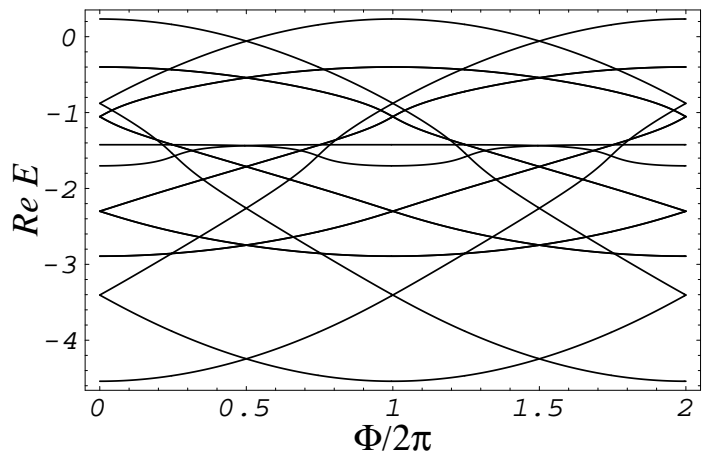

FIG. 4. The same spectral flow but with $\Psi=3$

in Fig. 5. If we start with the ground state at $\Theta=0$, and trace a path in $\Theta$ plane, we may have such spectral flows as depicted by several curves in this figure. For small $\Psi$ the path can be deformed to the real axis without changing the period. There exists, however, certain critical $\Psi_{\text {cr }}$ beyond which the gap between the first and the second excited states disappear. We denote it as a straight line in the figure. If we follow the path across this line, the period changes. It should be noted that the structure of Fig. 5 is periodic in $\Phi=2 \pi$, though we have drawn only the lines relevant to the flow of the ground state starting from $\Theta=0$. Therefore, paths on the $\Theta$ plane are divided into several classes classified in terms of the periods of the ground state. Although the spectral flow on the complex plane has quite rich structure, their essential property is understood by studying the typical case where the period of the ground state changes from $4 \pi$ to $8 \pi$. In the following section, we will estimate the corresponding critical twist $\Psi_{\text {cr }}$ based on the finite-size corrections.

Let us now check explicitly how the corresponding rapidities flow. The first example is Fig. 6 which corresponds to Fig. 2. Since we are concerned with the $N=6$ site system, the ground state is described by three rapidities sitting near the real axis at $\Phi=0$, indicated by dots. They continuously move on the complex plane, as $\Phi$ increases, towards the direction indicated by arrows. Finally at $\Phi=2 \pi$, rapidities reach the points

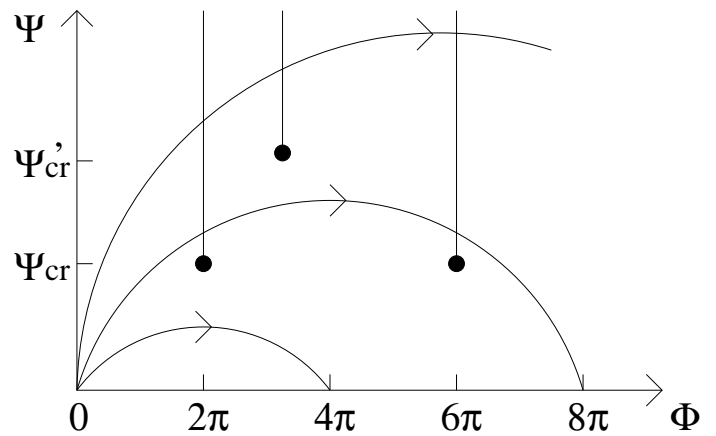

FIG. 5. Schematic illustration of the spectral flow of the ground state starting at $\Theta=0$. The ground state follows the curves towards the direction denoted by arrow and returns to the same ground state for the first time at the end of the curves.

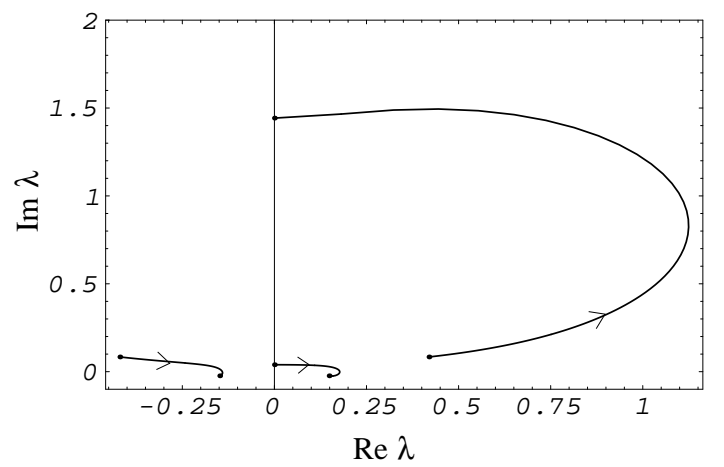

FIG. 6. Rapidity flow of the ground state of the $S^{z}=0$ sector with $\Psi=1.1$, corresponding to Fig. 2. Initial $(\Phi=0)$ and final $(\Phi=2 \pi)$ points are denoted by dots, flowing towards the direction indicated by arrows.

indicated by dots. What should be noted here is the motion of the last rapidity: After a long trip, it eventually reaches the imaginary axis. As a result, the whole distribution of the rapidities is changed considerably, but it is still symmetric with respect to the imaginary axis, which ensures that the total energy is real. Contrary to this, for $\Psi=1.2>\Psi_{\text {cr }}$ in Fig. 7 the final distribution is not symmetric, which suggests that there exists another state with the complex-conjugate energy corresponding to the configuration reflected with respect to the imaginary axis. We have checked that this is indeed true even for a larger system in Figs. 8 and 9. Therefore, we can see that whether the symmetric solution exists at $\Phi=2 \pi$ provides a criterion whether the gap exits. We have calculated $\Psi_{\mathrm{cr}}$ for several systems, summarized in Table 1 .

By using the criterion mentioned above, we wish to propose a practical method to estimate the critical value $\Psi_{\text {cr }}$ for a large but finite-size system. To this end let us assume that $\lambda_{M}$ sits on the imaginary axis. Then if $\Psi<\Psi_{\mathrm{cr}}$, the Bethe ansatz equations on this assumption have a solution, while for $\Psi>\Psi_{\text {cr }}$, they do not have a solution. Therefore, the critical value of $\Psi$ beyond which 


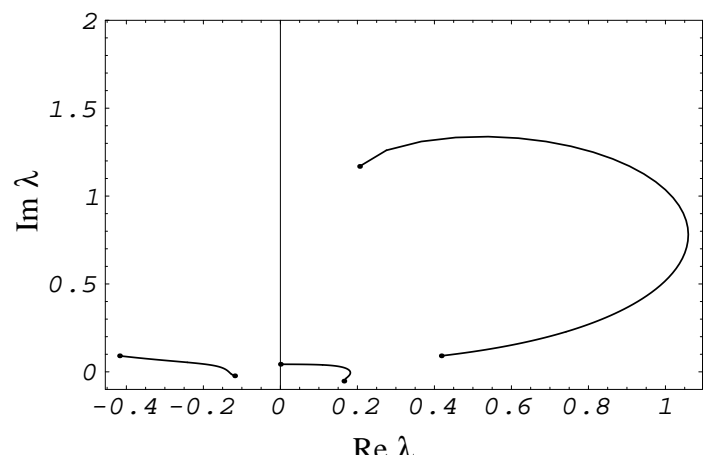

$\operatorname{Re} \lambda$

FIG. 7. The same rapidity flow but with $\Psi=1.2$, corresponding to Fig. 3

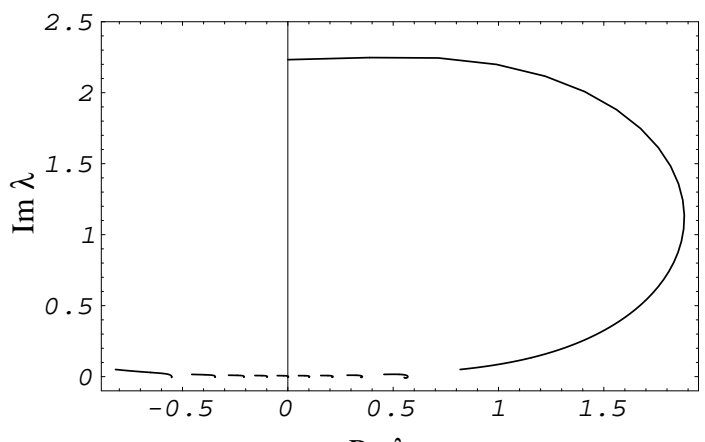

$\operatorname{Re} \lambda$

FIG. 8. The same rapidity flow but of $N=20$ system with $\Psi=0.7$

there exist such solutions can be identified with $\Psi_{\text {cr }}$. The problem becomes simpler if we put it in a different way: We have so far solved the Bethe ansatz equations for a given $\Psi$. We now set the problem for seeking for a possible $\Psi$ provided that the value of imaginary $\lambda_{M}$ is initially given. To solve this new problem, let us rewrite the Bethe ansatz equations at $\Phi=2 \pi$,

$$
\begin{gathered}
N p\left(\lambda_{j}\right)+\sum_{k \neq j}^{M-1} \theta\left(\lambda_{j}-\lambda_{k}\right)+\theta\left(i \lambda-\lambda_{k}\right) \\
=2 \pi\left(I_{j}+1\right)+i \Psi(\lambda),
\end{gathered}
$$

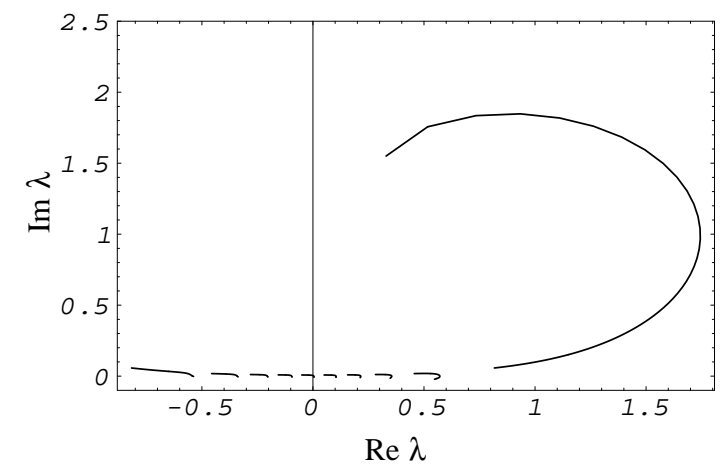

FIG. 9. The same rapidity flow but of $N=20$ system with $\Psi=0.8$

\begin{tabular}{c|llllll}
\hline \hline$N$ & 6 & 8 & 10 & 20 & 30 & 50 \\
\hline$\Psi_{\mathrm{cr}}$ & 1.1456 & 1.0225 & 0.9446 & 0.7655 & 0.6899 & 0.6141 \\
$\lambda_{M} / i$ & 1.208 & 1.308 & 1.381 & 1.622 & 1.759 & 1.915 \\
\hline$\Psi_{\max }$ & 1.0480 & 0.9532 & 0.8898 & 0.7363 & 0.6685 & 0.5991 \\
$\lambda$ & 1.372 & 1.446 & 1.507 & 1.712 & 1.837 & 1.999 \\
\hline$\Psi_{N, \max }$ & 1.1806 & 1.0536 & 0.9726 & 0.7861 & 0.7074 & 0.6287 \\
$\lambda$ & 1.323 & 1.398 & 1.461 & 1.673 & 1.802 & 1.967 \\
\hline \hline
\end{tabular}

TABLE I. The values $\Psi_{\text {cr }}$ calculated by several ways. " $\Psi_{\mathrm{cr}}$ " is the exact value calculated by the Bethe ansatz equations. " $\Psi_{\max }$ " is the maximum value of the function $\Psi(\lambda)$ in (3.2) by using the solution of Eq.(2.12). See III. B. " $\Psi_{N \text {, max }}$ " is the maximum value of the function $\Psi_{N}(\lambda)$ in (4.23).

$$
i \Psi(\lambda)=N p(i \lambda)+\sum_{k=1}^{M-1} \theta\left(i \lambda-\lambda_{k}\right)-2 \pi\left(I_{M}+1\right)
$$

In the above equations we should determine $\lambda_{j}(j=$ $1, \cdots, M-1)$ and $\Psi$ for a given real $\lambda$. The equations do not have a solution for $\Psi>\Psi_{\mathrm{cr}}$ so that for any given $\lambda$, $\Psi(\lambda)$ should not exceed $\Psi_{\mathrm{cr}}$. Namely, the function $\Psi(\lambda)$ should have a maximum value, which in turn gives $\Psi_{\mathrm{cr}}$. Therefore, the problem is now to determine the function $\Psi(\lambda)$, and find its maximum, which indeed allows us to obtain the critical twist $\Psi_{\mathrm{cr}}$.

\section{B. Bethe ansatz equations in a decoupling approximation}

Even if we employ the above trick, it is not so easy to determine $\Psi_{\mathrm{cr}}$. We here propose an approximate treatment to the problem and confirm that it works fairly well for $N=20$ system. Suppose that $N$ and $\lambda$ are large. Then the solutions of eq.(2.12) can be regarded as approximate solutions. Therefore, if we substitute them into eq.(3.2) and plot the result as a function of $\lambda$, extrapolating to the small $\lambda$ region, we have, for example, Fig. 10. This function indeed has a maximum value, which explains the existence of $\Psi_{\mathrm{cr}}$. It is remarkable that the maximum value thus obtained is quite close to the exact one in Table 1 . We list the maximum values of $\Psi(\lambda)$ and corresponding $\lambda$ in the same Table. They deviate from the exact value by only a few percent in general. Therefore, we can say that the value of $\Psi_{\text {cr }}$ is essentially determined by the rapidity $\lambda_{M}$ on the imaginary axis, and our approximate treatment works quite well.

In order to calculate $\Psi_{\text {cr }}$ self-consistently, we have to calculate the finite-size corrections based on eqs.(3.1) and (3.2). There are various $O(1 / N)$ terms, all of which should be taken into account. As we have already checked for the $N=20$ system, however, the contributions from the third term of the left-hand side and the second term 


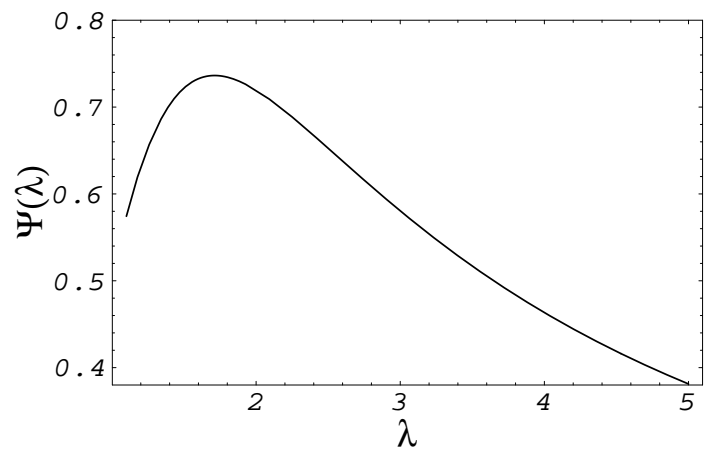

FIG. 10. Function $\Psi(\lambda)$ in eq.(3.2) for $N=20$ system. We use the solutions of eq. (2.12) as the nine rapidities $\lambda_{k}$ in this equation.

of the right hand side in eq.(3.1), i.e., $\theta\left(i \lambda-\lambda_{k}\right)$ and $i \Psi(\lambda)$, respectively, are quite small. Therefore, we set $\theta\left(i \lambda-\lambda_{k}\right) \rightarrow \pi$ and $i \Psi(\lambda) \rightarrow 0$ in this equation, and confirm the validity of this treatment by comparing the obtained results on this assumption with the exact $\Psi_{\text {cr }}$ for a small system. Thus, the Bethe equations for $\lambda_{j}$ $(j=1, \cdots, M-1)$ are decoupled from that for $i \lambda \equiv \lambda_{M}$, so that we use the notations $\mu_{j}$ for these $\lambda_{j}$ 's. In order to proceed the calculation, let us introduce the following functions from eqs.(2.12) and (3.3)

$Z_{N}(\mu)=\frac{p(\mu)}{2 \pi}+\frac{1}{2 \pi N} \sum_{k} \theta\left(\mu-\mu_{k}\right)$,

$i \Psi_{N}(\lambda)=N p(i \lambda)+\sum_{k} \theta\left(i \lambda-\mu_{k}\right)-\pi\left(\frac{N}{2}+1\right)$,

where $Z_{N}\left(\mu_{k}\right)=I_{k}^{\prime} / N$. All $\mu$ 's are on the real axis. The distribution function is given by

$$
\sigma_{N}(\mu)=\frac{p^{\prime}(\mu)}{2 \pi}+\frac{1}{2 \pi N} \sum_{k} \theta^{\prime}\left(\mu-\mu_{k}\right) .
$$

By definition,

$$
\int_{-\infty}^{\infty} d \mu \sigma_{N}(\mu)=\frac{1}{2}+\frac{1}{N}
$$

The above equations enable us to further proceed the analytic calculation, which will be performed separately in the next section.

\section{FINITE-SIZE CORRECTIONS}

We are now ready to evaluate finite-size corrections based on the equations in the preceding section. In particular, we derive the $\Psi_{\mathrm{cr}}$ as a maximum value of the function $\Psi(\lambda)$ in the bulk limit with leading finite-size corrections. So far, practical methods to compute the finite-size corrections for non-hermitian models [28,30] have not been well explored. So, we hope that the following treatment may also shed some light on such problems.

\section{A. Bulk limit}

We wish to first investigate Eqs.(3.4) and (3.3) in the bulk limit $N \rightarrow \infty$ to confirm that our approach is indeed consistent. Since the effects of the twist angle should be $O(1 / N)$, we expect that the function $\Psi(\lambda)$ in the bulk limit is identically zero for any $\lambda$, which is shown to be indeed satisfied in our treatment. In the bulk limit, Eqs.(3.4) and (3.3) are

$$
\begin{aligned}
& \sigma_{\infty}(\mu)=\frac{p^{\prime}(\mu)}{2 \pi}+\frac{1}{2 \pi} \int_{-\infty}^{\infty} d \mu^{\prime} \theta^{\prime}\left(\mu-\mu^{\prime}\right) \sigma_{\infty}\left(\mu^{\prime}\right), \\
& \frac{i \Psi_{\infty}(\lambda)}{N}=p(i \lambda)+\int_{-\infty}^{\infty} d \mu \theta(i \lambda-\mu) \sigma_{\infty}(\mu)-\frac{\pi}{2},
\end{aligned}
$$

where the left-hand side of the second equation means $\lim _{N \rightarrow \infty} \frac{i \Psi_{N}(\lambda)}{N}$. Since $\mu$ 's are distributed on the real axis, their distribution can be easily determined by the Fourier transformation. The result is

$$
\sigma_{\infty}(\mu)=\frac{1}{2} \operatorname{sech} \pi \mu
$$

By substituting this solution into eq.(4.2), we have

$$
\begin{aligned}
\frac{i \Psi_{\infty}(\lambda)}{N}= & i \int_{-\infty}^{\infty} d \mu \frac{\operatorname{sech} \pi \mu}{2} \ln \left(\frac{\mu-i(\lambda-1)}{\mu-i(\lambda+1)}\right) \\
& -i \ln \left(\frac{\lambda-1 / 2}{\lambda+1 / 2}\right),
\end{aligned}
$$

where we have assumed $\lambda>1$. The first term of the righthand side is evaluated by extending the contour integral in the lower half plane and summing up the infinite series of residues on the imaginary axis. We find that it cancels the second term, and leads to

$$
\frac{i \Psi_{\infty}(\lambda)}{N}=0 .
$$

This proves that our treatment indeed produces the correct result in the bulk limit because effects of the twist should disappear in this limit.

\section{B. $1 / N$ corrections}

So far we have checked that $\Psi_{\infty}(\lambda)=0$ for any $\lambda>1$, which means that the gap in the spectrum always disappears in the bulk limit. It is quite natural because the gap is caused by marginally irrelevant interaction. Now we proceed to finite-size systems, for which we expect a finite $\Psi_{\mathrm{cr}}$.

Subtracting eq. (4.1) from eq.(3.4) we have the identity valid for a system with arbitrary $N$,

$$
\begin{aligned}
\sigma_{N}(\mu)-\sigma_{\infty}(\mu)= & -\int_{-\infty}^{\infty} d \mu^{\prime} q\left(\mu-\mu^{\prime}\right) \\
& \times\left[\frac{1}{N} \sum_{k} \delta\left(\mu^{\prime}-\mu_{k}\right)-\sigma_{N}\left(\mu^{\prime}\right)\right],
\end{aligned}
$$


where the kernel $q(\mu)$ is

$$
q(\mu)=\frac{1}{2 \pi} \int_{-\infty}^{\infty} d \omega e^{-i \omega \mu} \frac{1}{e^{|\omega|}+1} .
$$

Denote the largest rapidity on the real axis as $\Lambda$. Then $Z_{N}(\Lambda)=\frac{1}{4}-\frac{1}{N}$, or equivalently,

$$
\int_{-\Lambda}^{\Lambda} d \mu \sigma_{N}(\mu)=\frac{1}{2}-\frac{2}{N}
$$

Similarly, $\Psi(\lambda)$ is rewritten as

$$
\begin{aligned}
\frac{i \Psi_{N}(\lambda)}{N}= & \int_{-\infty}^{\infty} d \mu Q(\lambda, \mu) \\
& \times\left[\frac{1}{N} \sum_{k} \delta\left(\mu-\mu_{k}\right)-\sigma_{N}(\mu)\right]-\frac{\pi}{N},
\end{aligned}
$$

where

$$
Q(\lambda, \mu)=\theta(i \lambda-\mu)-\int_{-\infty}^{\infty} d \mu^{\prime} \theta\left(i \lambda-\mu^{\prime}\right) q\left(\mu^{\prime}-\mu\right),
$$

The kernel $Q(\lambda, \mu)$ can be evaluated by counter integral in the lower half plain, yielding the result

$$
\begin{aligned}
& Q(\lambda, \mu)=-\frac{\pi}{2}+\widetilde{Q}(\lambda, \mu), \\
& \widetilde{Q}(\lambda, \mu)=i \ln \left(\frac{\mu-i(\lambda-1)}{\mu-i \lambda}\right) .
\end{aligned}
$$

Substituting this equation into Eq.(4.9), we find that the first constant term in Eq. 4.11) cancels the last constant term in Eq.4.9). Therefore,

$$
\begin{aligned}
\frac{i \Psi_{N}(\lambda)}{N}= & \int_{-\infty}^{\infty} d \mu \widetilde{Q}(\lambda, \mu) \\
& \times\left[\frac{1}{N} \sum_{k} \delta\left(\mu-\mu_{k}\right)-\sigma_{N}(\mu)\right] .
\end{aligned}
$$

To evaluate the leading finite-size effects, let us apply the Euler-Maclaurin formula to Eqs.(4.6) and (4.9). Up to $O\left(N^{-2}\right)$, we have

$$
\begin{gathered}
\sigma_{N}(\mu)-\sigma_{\infty}(\mu)=\int_{\Lambda}^{\infty} d \mu^{\prime} q\left(\mu-\mu^{\prime}\right) \sigma_{N}\left(\mu^{\prime}\right) \\
-\frac{q(\mu-\Lambda)}{2 N}+\frac{q^{\prime}(\mu-\Lambda)}{12 N^{2} \sigma_{N}(\Lambda)}+(-\Lambda \text { terms }), \\
\frac{i \Psi_{N}(\lambda)}{N}=-2 \int_{\Lambda}^{\infty} d \mu \widetilde{Q}(\lambda, \mu) \sigma_{N}(\mu) \\
+\frac{\widetilde{Q}(\lambda, \Lambda)}{N}+\frac{\widetilde{Q}^{\prime}(\lambda, \Lambda)}{6 N^{2} \sigma_{N}(\Lambda)}
\end{gathered}
$$

where $\widetilde{Q}^{\prime}(\lambda, \Lambda)=\partial \widetilde{Q}(\lambda, \Lambda) / \partial \Lambda$. The cut-off $\Lambda$ is determined by the condition

$$
\int_{\Lambda}^{\infty} d \mu \sigma_{N}(\mu)=\frac{3}{2 N}
$$

\section{Finite size corrections to $\sigma_{N}(\mu)$}

As we have exploited the decoupling approximation, we can evaluate the finite-size corrections to $\sigma_{N}(\lambda)$ following standard techniques 31 34]. Introducing

$$
\begin{aligned}
& \sigma_{N}^{+}(\mu)= \begin{cases}\sigma_{N}(\mu+\Lambda) & \text { for } \quad \mu>0 \\
0 & \text { for } \quad \mu<0,\end{cases} \\
& \sigma_{N}^{-}(\mu)= \begin{cases}0 & \text { for } \quad \mu>0 \\
\sigma_{N}(\mu+\Lambda) & \text { for } \quad \mu<0,\end{cases}
\end{aligned}
$$

we can write down eq.(4.13) in the region $\mu \sim \Lambda$, by neglecting $-\Lambda$ terms,

$$
\begin{array}{r}
\sigma_{N}^{+}(\mu)+\sigma_{N}^{-}(\mu)-\int_{-\infty}^{\infty} d \mu^{\prime} q\left(\mu-\mu^{\prime}\right) \sigma_{N}^{+}\left(\mu^{\prime}\right) \\
=\sigma_{\infty}(\mu+\Lambda)-\frac{q(\mu)}{2 N}+\frac{q^{\prime}(\mu)}{12 N^{2} \sigma_{N}(\Lambda)} .
\end{array}
$$

Solving the equation in a standard way summarized briefly in the appendix, we end up with the final results

$$
\tilde{\sigma}_{N}^{+}(\omega)=C(\omega)+G_{+}(\omega / 2 \pi)\left[P(\omega)+Q_{+}(\omega)\right],
$$

where $\widetilde{\sigma}_{N}^{ \pm}(\omega)$ is the Fourier-transformation of $\sigma_{N}^{ \pm}(\lambda)$ and where

$$
\begin{aligned}
& C(\omega)=\frac{1}{\sqrt{2 \pi}}\left(\frac{1}{2 \pi}+\frac{i \omega}{12 N^{2} \sigma_{N}(\Lambda)}\right), \\
& G_{+}(\omega / 2 \pi)=\sqrt{2 \pi}\left(-\frac{i \omega}{2 \pi e}\right)^{-\frac{i \omega}{2 \pi}} / \Gamma\left(\frac{1}{2}-\frac{i \omega}{2 \pi}\right), \\
& P(\omega)=-\frac{1}{\sqrt{2 \pi}}\left(\frac{1}{2 N}-\frac{2 \pi i g}{12 N^{2} \sigma_{N}(\Lambda)}+\frac{i \omega}{12 N^{2} \sigma_{N}(\Lambda)}\right), \\
& Q_{+}(\omega)=\frac{i}{\sqrt{2 \pi}} \frac{e^{-\pi \Lambda}}{\omega+\pi i} G_{+}(i / 2),
\end{aligned}
$$

with

$$
\begin{aligned}
& i g=1 / 24, \\
& \sigma_{N}(\Lambda)=\frac{\pi}{N} \alpha, \\
& \Lambda=\frac{1}{\pi} \ln \left(\frac{N}{\sqrt{\pi e} \beta}\right) .
\end{aligned}
$$

Here several constants are given by

$$
\begin{aligned}
& \alpha=\frac{1}{2}\left(a_{0}+\sqrt{a_{0}^{2}-4 a_{1}}\right), \\
& \beta=\frac{1}{2}+\frac{1}{\sqrt{2}}-\frac{i g}{6 \alpha}, \\
& a_{0}=\frac{1}{2}+\frac{1}{\sqrt{2}}+i g, \\
& a_{1}=\frac{i g+(i g)^{2}}{6} .
\end{aligned}
$$




\section{Finite-size corrections to $\Psi_{N}(\lambda)$}

By using the preceding results for $\sigma_{N}(\lambda)$, let us now calculate $\Psi_{N}(\lambda)$ with finite-size effects. To begin with, we convert the first term in the right-hand side of eq.4.14) into the integral in Fourier space,

$$
\begin{aligned}
& -2 \int_{\Lambda}^{\infty} d \mu \widetilde{Q}(\lambda, \mu) \sigma_{N}(\mu) \\
& =-i \sqrt{2 \pi} \int_{-\infty}^{\infty} d \omega e^{i \Lambda \omega} \frac{e^{-\lambda|\omega|}-e^{-(\lambda-1)|\omega|}}{|\omega|} \widetilde{\sigma}_{N}^{+}(\omega) .
\end{aligned}
$$

It is easily shown that $C(\omega)$ in $\tilde{\sigma}_{N}^{+}(\omega)$ cancels out the second and third terms in the right-hand side of eq.(4.14). Therefore, we eventually arrive at

$$
\begin{aligned}
\Psi_{N}(\lambda)= & -\int_{-\infty}^{\infty} d \omega e^{i \Lambda \omega} \frac{e^{-\lambda|\omega|}-e^{-(\lambda-1)|\omega|}}{|\omega|} \\
& \times G_{+}(\omega / 2 \pi)\left(P(\omega)+Q_{+}(\omega)\right) .
\end{aligned}
$$

We have evaluated the integral numerically for the $N=20$ system in Fig. 11, corresponding to Fig. 10. As we expect, the leading finite-size calculation yields the desirable results. In fact, the maximum value of this

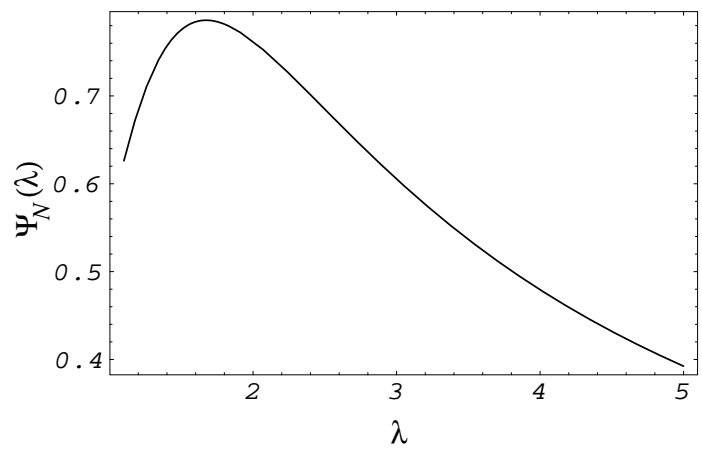

FIG. 11. $\Psi_{N}(\lambda)$ for $N=20$ system

function reproduces the critical $\Psi$ expected for small systems fairly well, which are listed in Table If.

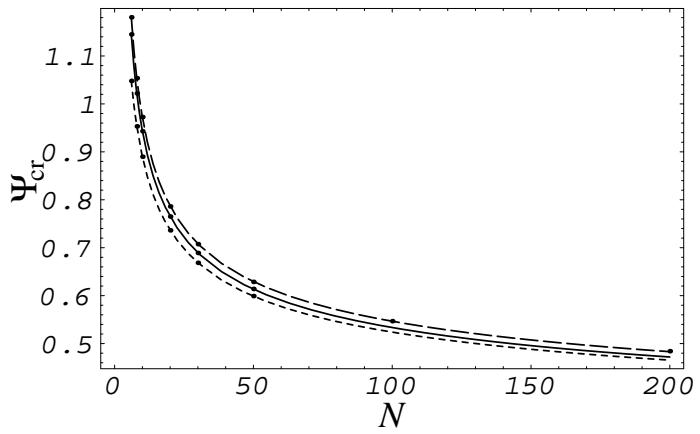

FIG. 12. The data of $\Psi_{\text {cr }}$ in Table 1 and their least-square fits. See the text.

It seems not easy to precisely determine the large$N$ behavior of the function $\Psi(\lambda)$. However, observing the data for several small $N$ systems of the exact
Bethe ansatz calculation and also for larger $N$ systems of Eq. (4.23), we can predict $1 / \ln N$ behavior in the asymptotic regime. To confirm this explicitly, we plot in Fig. 12 the least-square fit using $(\ln N)^{-n}$ with $n=1,2,3$ with the data points. The solid-line is for the exact Bethe ansatz results, while the dotted- and dashed-lines are for the decoupled Bethe ansatz results of finite-size systems in section III. B. and for Eq. (4.23), respectively. As expected, the larger the system is, the smaller the difference between the dotted- and dashed-lines becomes. Furthermore, these $\Psi_{\mathrm{cr}}$ in a decoupling approximation are very close to the exact $\Psi_{\mathrm{cr}}$. Note that $\Psi_{\mathrm{cr}}$ is not a gap itself between the first and second excited states. The gap of $O(1 / N)$ exist there, and we need $\Psi_{\text {cr }}$ of $O(1 / \ln N)$ to close this gap.

\section{SUMMARY AND DISCUSSIONS}

Stimulated by the recent advance in the theory for localization-delocalization transitions in 1D nonhermitian systems, we have studied how the nonhermitian property affects the integrable Heisenberg spin model. In particular, we have focused the particular attention on the spectral flow by imposing twisted boundary conditions with complex twist. By solving the Bethe ansatz equations numerically for finite systems, we have shown that a global structure of the spectral flow completely changes its character as the imaginary twist increases. Namely, the period of the ground state increases from $4 \pi$ to $8 \pi$ at a certain imaginary twist angle. If the imaginary twist is further increased, the period successively jumps to a higher value at the corresponding imaginary twist angle. The origin of this phenomenon has been clarified: the small $1 / N$ energy gaps for a finite system caused by irrelevant interactions are closed by the non-hermitian hopping of XY term. To determine the critical imaginary twist for the transition, we have also proposed a practical method to evaluate the finitesize corrections, and discussed the behavior in the large $N$ regime. Although we have neglected some terms which may give $O(1 / N)$ contributions in this treatment, the estimated value of $\Psi_{\mathrm{cr}}$ is quite close to the exact one. Since practical ways to explicitly evaluate the finite-size corrections for non-hermitian models have not been developed well, the present treatment may provide a guideline to study such problems in more detail.

In this paper we have treated $\Phi$ and $\Psi$ on an equal footing. Before concluding the paper, we briefly make a comment on the physical origin of these twists. As mentioned in the introduction, the real twist $\Phi$ can be naturally regarded as an Aharonov-Bohm flux. As for imaginary $\Psi$, its origin may be ascribed to randomness in the system. This is indeed seen by observing the Hamiltonian, 


$$
H=\sum_{j}\left[\frac{1}{2}\left(e^{-\Psi_{j}} S_{j}^{+} S_{j+1}^{-}+e^{\Psi_{j}} S_{j}^{-} S_{j+1}^{+}\right)+S_{j}^{z} S_{j+1}^{z}\right]
$$

with the boundary condition

$$
S_{j+N}^{ \pm}=e^{ \pm i \Phi} S_{j}, \quad S_{j+N}^{z}=S_{j}^{z} .
$$

where $\Psi_{j}$ are not necessarily uniform. The Bethe ansatz equation is the same as Eq.(2.6), but with

$$
\Theta=\Phi+i \sum_{j} \Psi_{j}
$$

Therefore, it is seen that our imaginary twist can be regarded as the consequence of non-hermitian random hoppings. By taking into account the ensemble average over random variables $\Psi_{j}$, we may have a chance to discuss the effects of random non-hermitian hoppings, which should be treated in the future work.

The Hamiltonian (5.1) is also useful to see how the present analysis is related to the metal-insulator transition in the asymmetric models, and also to the delocalization transition in the Hatano-Nelson model. To this end, let us set $\Psi_{j}=\Psi / N$, where $\Psi$ is given by (2.3). By using the hard-core boson representation, the $\mathrm{XY}$ term is converted into the hopping term of bosons, $H_{\text {hop }}=\sum_{j}\left(e^{-\Psi / N} b_{j}^{\dagger} b_{j+1}+e^{\Psi / N} b_{j+1}^{\dagger} b_{j}\right)$, where $b_{j}$ is a boson operator. Namely, the present model is equivalent to the interacting bosonic lattice model with an imaginary vector potential. Note that the kinetic part is essentially the same as that for the Hatano-Nelson model. For the latter model, Hatano and Nelson demonstrated that the "insulating" state caused by randomness can be delocalized and become metallic at a certain critical $\Psi$ when the strength of the imaginary vector potential is increased. This implies that the transition is caused by the competition between the imaginary vector potential and the random potential. We can also observe a similar insulator-metal transition caused by imaginary vector potential for the periodic model such as the asymmetric XXZ model with Ising-type anisotropy [29], for which the massive (insulating) state is formed by the many-body interaction, instead of randomness. In contrast to the above examples, for the isotropic Heisenberg model (5.1) we have dealt with, the system is massless and "metallic" in the thermodynamic limit even for $\Psi=0$. However, we should recall that there still exist small (mesoscopic) gaps formed by irrelevant many-body interactions for a finite-size system, which control the period of the spectral flow for the ground state. We have shown explicitly via the present study that the competition between the imaginary vector potential and the many-body interaction indeed modifies the period, which implies that the mesoscopic gaps are closed by the imaginary vector potential: Our transition is characterized by the collapse of the mesoscopic gaps, whereas that for the $\mathrm{XXZ}$ case is by the collapse of the ordinary gap. We can now see that there are deep relationship among the transition found in the spectral flow of the present model, the "insulatormetal" transition for the XXZ model, and the HatanoNelson model, although at first sight our results may not have any essential connections to latter phenomena; they may indeed share some essential features, e.g., the transition is driven by imaginary vector potentials and is characterized by complex eigenvalues at criticality. It may be interesting to study wider class of models with imaginary vector potentials to understand a novel class of the metal-insulator transition in a unified framework.

\section{ACKNOWLEDGMENTS}

The authors would like to thank H.-P. Eckle, A. Klümper and M. Chiba for valuable discussions. Numerical computation in this work was carried out at the Yukawa Institute Computer Facility. This work is partly supported by the Grant-in-Aid from the Ministry of Education, Science and Culture, Japan.

\section{APPENDIX A: WIENER-HOPF EQUATION}

Since there exist extensive studies of finite-size calculations 31 34], we summarize it briefly for reference sake.

Introducing the Fourier transformation with the normalization

$$
\sigma_{N}^{ \pm}(\lambda)=\frac{1}{\sqrt{2 \pi}} \int_{-\infty}^{\infty} d \omega e^{-i \lambda \omega} \widetilde{\sigma}_{N}^{ \pm}(\omega),
$$

Eq. (4.17) is given by the Fourier component

$$
\widetilde{\sigma}_{N}^{-}(\omega)+\frac{\widetilde{\sigma}_{N}^{+}(\omega)-C(\omega)}{e^{-|\omega|}+1}=\frac{e^{-i \omega \Lambda} \operatorname{sech} \frac{\omega}{2}}{2 \sqrt{2 \pi}}-C(\omega)
$$

The kernel is now factorized as

$$
e^{-|\omega|}+1=G_{+}(\omega / 2 \pi) G_{-}(\omega / 2 \pi),
$$

where $G_{ \pm}(z)$ is holomorphic in the upper-(lower) half plain, respectively. The asymptotic form is

$$
G_{+}(z)=1+\frac{g}{z}+\frac{g^{2}}{2 z^{2}}+O\left(z^{-3}\right) .
$$

Then Eq.(A2) can be written as

$$
\begin{aligned}
& \frac{\tilde{\sigma}_{N}^{+}(\omega)-C(\omega)}{G_{+}(\omega / 2 \pi)}-Q_{+}(\omega) \\
& \quad=-G_{-}(\omega / 2 \pi)\left(\widetilde{\sigma}_{N}^{-}(\omega)+C(\omega)\right)+Q_{-}(\omega) \\
& \quad \equiv P(\omega) .
\end{aligned}
$$


where $Q_{ \pm}(\omega)$ is holomorphic in the upper-(lower) half plain, satisfying

$$
G_{-}(\omega / 2 \pi) \frac{e^{-i \omega \Lambda} \operatorname{sech} \frac{\omega}{2}}{2 \sqrt{2 \pi}}=Q_{+}(\omega)+Q_{-}(\omega)
$$

where we take into account only the contribution from the pole nearest to the real axis in the text. From the asymptotic form Eq.(A4), $P(\omega)$ is determined, which is summarized in the text.

The parameters $\Lambda$ and $\sigma(\Lambda)$ are determined by the conditions Eq. (4.15) and

$$
\sigma_{N}(\Lambda)=\frac{2}{\sqrt{2 \pi}} \int_{-\infty}^{\infty} d \omega \widetilde{\sigma}_{N}^{+}(\omega)
$$

or more precisely,

$$
\begin{aligned}
& G_{+}(i / 2) e^{-\pi \Lambda}=\left(\frac{1}{2}+\frac{1}{\sqrt{2}}\right) \frac{\pi}{N}-\frac{\pi(2 \pi i g)}{12 N^{2} \sigma_{N}(\Lambda)}, \\
& \sigma_{N}(\Lambda)=G_{+}(i / 2) e^{-\pi \Lambda}+\frac{(2 \pi i g)}{2 N}-\frac{(2 \pi i g)^{2}}{24 N^{2} \sigma_{N}(\Lambda)} .
\end{aligned}
$$

The solutions are summarized in the text.

* Email: fukui@yukawa.kyoto-u.ac.jp

[1] H. J. de Vega, Nucl. Phys. B240 (1984) 495.

[2] F. C. Alcaraz, M. N. Barber and M. T. Batchelar, Ann. Phys. 182 (1988) 280.

[3] C. M. Yung and M. T. Batcherlor, Nucl. Phys. B446 (1995) 461.

[4] V.E. Korepin, N.M. Bogoliubov and A.G. Izergin, "Quantum Inverse Scattering Method and Correlation Functions", Cambridge University Press (1993).

[5] N. Byers and C. N. Yang, Phys. Rev. Lett. 7 (1961) 46.

[6] W. Kohn, Phys. Rev. 133 (1964) 171.

[7] B.S. Shastry and B. Sutherland, Phys. Rev. Lett. 65 (1990) 243.

[8] B. Sutherland and B. S. Shastry, Phys. Rev. Lett. 65 (1990) 1833.

[9] N. Yu and M. Fowler, Phys. Rev. 46 (1992) 14583.

[10] R.A. Römer, H.-P. Eckle and B. Sutherland, Phys. Rev. B52 (1995) 1656.

[11] B. Sutherland, Phys. Rev. Lett. 74 (1995) 816.

[12] K. Kusakabe and H. Aoki, J. Phys. Soc. Jpn 65 (1996) 2772.

[13] V. E. Korepin and A. C. T. Wu, Int. J. Mod. Phys. B5 (1991) 497.

[14] N. Fumita, H. Itoyama and T. Oota, Int. J. Mod. Phys. A12 (1997) 801.

[15] T. Fukui and N. Kawakami, J. Phys. Soc. Jpn 65 (1996) 2824.

[16] T. Fukui and N. Kawakami, Phys. Rev. Lett. 76 (1996) 4242; Phys. Rev. B54 (1996) 5346; Nucl. Phys. B483 (1997) 663.
[17] J. T. Liu and D. F. Wang, Phys. Rev. B55 (1997) R3344; Phys. Rev. B56 (1997) 2312.

[18] F. D. M. Haldane, Phys. Rev. Lett. 67 (1991) 937.

[19] A. Kitazawa, J. Phys. A30 (1997) L285.

[20] T. Nassar and O. Tirkkonen, hep-th/9707098.

[21] N. Hatano and D. R. Nelson, Phys. Rev. Lett. 77 (1997) 570; cond-mat/9705290.

[22] C. P. Yang, Phys. Rev. Lett. 19 (1967) 586; B. Sutherland, C. N. Yang and C. P. Yang, Phys. Rev. Lett. 19 (1967) 588.

[23] I. M. Nolden, J. Stat. Phys. 67 (1992) 155.

[24] D. J. Bukman and J. D. Shore, J. Stat. Phys. 78 (1995) 1277.

[25] L. H. Gwa and H. Spohn, Phys. Rev. A46 (1993) 844.

[26] F. C. Alcaraz, H. Droz, M. Henkel and V. Rittenberg, Ann. Phys. 230 (1994) 250.

[27] J. Neergaard and M. den Nijs, Phys. Rev. Lett. 74 (1995) 730.

[28] D. Kim, Phys. Rev. E52 (1995) 3512.

[29] G. Albertini, S. R. Dahmen and B. Wehefritz, J. Phys. A29 (1996) L369; Nucl. Phys. B493 (1997) 541.

[30] J. D. Noh and D. Kim, cond-mat/9511001.

[31] H. J. de Vega and F. Woynarovich, Nucl. Phys. B251 (1985) 439.

[32] F. Woynarovich and H.-P. Eckle, J. Phys. A20 (1987) L97.

[33] F. Woynarovich, H.-P. Eckle and T. T. Truong, J. Phys. A22 (1989) 4027.

[34] C. J. Hamer, G. R. W. Quispel and M. T. Batchelor, J. Phys. A20 (1987) 5677. 